\title{
Waves in Flexural Beams with Nonlinear Adhesive Interaction
}

\author{
G. M. Coclite®, G. Devillanova®, and F. Maddalena®
}

\begin{abstract}
The paper studies the initial boundary value problem related to the dynamic evolution of an elastic beam interacting with a substrate through an elastic-breakable forcing term. This discontinuous interaction is aimed to model the phenomenon of attachment-detachment of the beam occurring in adhesion phenomena. We prove existence of solutions in energy space and exhibit various counterexamples to uniqueness. Furthermore we characterize some relevant features of the solutions, ruling the main effects of the nonlinearity due to the elastic-breakable term on the dynamical evolution, by proving the linearization property according to Gérard (J Funct Anal 141(1):60-98, 1996) and an asymptotic result pertaining the long time behavior.
\end{abstract}

Mathematics Subject Classification. 35L05, 74B20, 35J25.

Keywords. Beam equation, Discontinuous interaction, Flexural waves.

\section{Introduction}

In a broader sense the term adhesion refers to a physical situation in which two material bodies, during their mechanical evolution, experience a contact interaction which fails in (possibly bounded) regions of space-time and restores after a while. The phenomenon strongly depends on the constitutive properties of the involved materials and a crucial problem relies in understanding the nature of the interaction. The manifestation of such phenomenon occurs at every scale, ranging from DNA molecules to the structural engineering works. Mathematics has looked at these problems, at least in the stationary case, since the seminal paper [1] and in some

The authors are members of the Gruppo Nazionale per l'Analisi Matematica, la Probabilità e le loro Applicazioni (GNAMPA) of the Istituto Nazionale di Alta Matematica (INdAM). The second author is also supported by MIUR-FFABR-2017 research grant. The authors have been partially supported by the Research Project of National Relevance "Multiscale Innovative Materials and Structures" granted by the Italian Ministry of Education, University and Research (MIUR Prin 2017, project code 2017J4EAYB and the Italian Ministry of Education, University and Research under the Programme Department of Excellence Legge 232/2016 (Grant No. CUP-D94I18000260001). 
recent works (see, e.g., [7-11]) one of the authors contributed to the study of the static problem of adhesion of elastic structures by exploiting different constitutive assumptions to the aim of characterizing, in a variational framework, the interplay of the debonding with other constitutive properties.

However the dynamical problem is a different story since at the heart of the question stays the understanding of the attachement-detachement occurrence and how this affects the whole evolution problem. This is a subtle problem since the analytical tools at disposal, such as spatio-temporal estimates in some norms, seem too rough to catch exhaustive quantitative informations, even in short time.

In $[3,4]$ the simplest mechanical model consisting in elastic string was considered, whereas a discontinuous forcing term was assumed to model the adhesive interaction of the string with a rigid substrate. The resulting mathematical problem is then ruled by an initial boundary value problem for a semilinear second order wave equation and the results in $[3,4]$ show some tricky peculiarities of the problem. As it is well known in continuum mechanics, the other basic model for one dimensional elastic structures is represented by the so called Bernoulli-Navier beam governing the flexural deformations of a slender material body. In the linear elastic framework the equation expressing the balance of momentum is ruled by a fourth order spatial differential operator. Analogously to [3], we assume a discontinuous forcing term to model the adhesive interaction of the beam with the external environment. One can visualize as a physical situation an elastic beam connected to a rigid substrate through a foundation made of continuous distribution of elastic-breakable springs.

We study the well posedness of the mathematical problem proving existence of global in time solutions in the natural energy space and exploit some features of the solutions to obtain information about the role played by the attachment-detachment occurrence on the dynamical evolution. Indeed, in [2] it was proved that the main effects induced by the nonlinearity at the transition from attached to detached states consist in a loss of regularity of the solution and in a migration of the total energy through the scales. Here we deepen the analysis by using the linearization condition introduced by Gérard in [5] according to which one can conclude that if the semilinear evolution problem satisfies such condition, then the nonlinear forcing term does not induce any new oscillation or energy concentrations [5]. Furthermore we prove an asymptotic result for the long time behavior in the case of bounded solutions, asserting the occurrence of three mutually exclusive states: the trivial one, the totally detached state, the totally attached state.

The paper is organized as follows. In Sect. 2 we formulate the initial boundary value problem. In Sect. 3 we state the main results of the paper consisting in Theorem 3.2 (Existence of solutions), Theorem 3.3 (Characerization of adhesive states), Theorem 3.4 (Long time behavior), Theorem 3.5 (Linearization property). The proofs of these theorems are given respectively in Sects. 4, 5, 6, 7. In Sect. 8 we provide some examples showing non-uniqueness and lack of smoothness of the solutions. 


\section{Statement of the Problem}

Let us consider an elastic beam under Bernoulli-Navier constitutive assumption, occupying in the reference configuration the interval $[0, L] \subset \mathbb{R}$, the balance of linear momentum delivers the semilinear initial boundary value problem

$$
\begin{cases}\rho \partial_{t t}^{2} u=-\mu \partial_{x x x x}^{4} u-\Phi^{\prime}(u) & t>0,0<x<L, \\ \partial_{x x}^{2} u(t, 0)=\partial_{x x}^{2} u(t, L)=0 & t>0, \\ \partial_{x x x}^{3} u(t, 0)=\partial_{x x x}^{3} u(t, L)=0 & t>0, \\ u(0, x)=u_{0}(x) & 0<x<L, \\ \partial_{t} u(0, x)=u_{1}(x) & 0<x<L .\end{cases}
$$

We shall assume that

(H.1) $\Phi \in C(\mathbb{R}) \cap C^{2}(\mathbb{R} \backslash\{1,-1\}), \Phi$ is positive, constant in $(-\infty,-1]$ and in $[1, \infty)$, convex in $[-1,1]$, decreasing in $[-1,0]$, increasing in $[0,1]$, and $\Phi(u) \geq \kappa u^{2}$ in $[-1,1]$ for some constant $\kappa>0$;

(H.2) $u_{0} \in H^{2}(0, L), u_{1} \in L^{2}(0, L)$;

(H.3) $\rho>0$ is the constant material density and $\mu>0$ is the elastic stiffness.

As a consequence of (H.1), $\Phi^{\prime}$ has a jump discontinuity in $u= \pm 1$ and

$$
\begin{aligned}
u \in(-\infty,-1) \cup(1, \infty) & \Rightarrow \Phi^{\prime}(u)=0, \\
0<u<1 & \Rightarrow 0<\Phi^{\prime}(u) \leq \lim _{u \rightarrow 1^{-}} \Phi^{\prime}(u), \\
-1<u<0 & \Rightarrow 0>\Phi^{\prime}(u) \geq \lim _{u \rightarrow-1^{+}} \Phi^{\prime}(u) .
\end{aligned}
$$

The set $\{|u|=1\}$ of these discontinuities separates the attached zone $\{|u|<1\}$ from the detached one $\{|u|>1\}$ since the forcing term $\Phi^{\prime}(u)$ is thought to model the elastic breakable interaction between the beam and the external environment. Such forcing term confers to the problem a localized nonlinearity which affects the evolution in a significant way.

To fix ideas, a function satisfying (H.1) is

$$
\Phi(u)=\left\{\begin{array}{lll}
u^{2} & \text { if } & |u| \leq 1 \\
1 & \text { if } & |u|>1
\end{array}\right.
$$

In particular we have for all $u \neq \pm 1$

$$
\Phi^{\prime}(u)=\left\{\begin{array}{lll}
2 u & \text { if } & |u|<1 \\
0 & \text { if } & |u|>1
\end{array}\right.
$$

The natural energy associated to the Problem (2.1) (i.e. to any solution $u$ to (2.1)), is given at time $t$ by the quantity

$$
E[u](t)=\int_{0}^{L}\left(\frac{\rho\left(\partial_{t} u(t, x)\right)^{2}+\mu\left(\partial_{x x}^{2} u(t, x)\right)^{2}}{2}+\Phi(u(t, x))\right) d x
$$

In general, the lack of Lipschitz continuity in the nonlinear term $\Phi^{\prime}$ suggests we cannot expect the existence of conservative solutions, i.e. solutions that preserve energy. Also the physics underlying the problem, foresees a kind of dissipation when 
the material is detaching from the substrate and this is accompanied by hysteresis cycles (see e.g. [8, Appendix B]).

\section{Main Results}

We begin by studying the well-posedness of Problem (2.1) and the regularity of its solutions (see Definition 3.1 below). We prove the existence of Lipshitz continuous dissipative solutions and give examples of distinct solutions to (2.1) which do not depend continuously on the initial conditions.

Definition 3.1. We say that a function $u:[0, \infty) \times[0, L] \rightarrow \mathbb{R}$ is a dissipative solution of (2.1) if

(i) $u \in C([0, \infty) \times[0, L])$;

(ii) $\partial_{t} u, \partial_{x x}^{2} u \in L^{\infty}\left(0, \infty ; L^{2}(0, L)\right)$;

(iii) $u$ is a weak solution to (2.1), i.e. for every test function $\varphi \in C^{\infty}\left(\mathbb{R}^{2}\right)$ with compact support

$$
\begin{aligned}
\int_{0}^{\infty} & \int_{0}^{L}\left(\rho u \partial_{t t}^{2} \varphi+\mu \partial_{x x}^{2} u \partial_{x x}^{2} \varphi+h_{u} \varphi\right) d t d x \\
& -\int_{0}^{L} \rho u_{1}(x) \varphi(0, x) d x+\int_{0}^{L} \rho u_{0}(x) \partial_{t} \varphi(0, x) d x=0
\end{aligned}
$$

where $h_{u} \in \partial \Phi(u)$, that is the subdifferential of $\Phi$ at $u$;

(iv) $u$ may dissipate energy, i.e. for almost every $t>0: E[u](t) \leq E[u](0)$, namely $($ see $(2.4))$

$$
\begin{aligned}
E[u](t) & =\int_{0}^{L}\left(\frac{\rho\left(\partial_{t} u(t, x)\right)^{2}+\mu\left(\partial_{x x}^{2} u(t, x)\right)^{2}}{2}+\Phi(u(t, x))\right) d x \\
& \leq \int_{0}^{L}\left(\frac{\rho\left(u_{1}(x)\right)^{2}+\mu\left(\partial_{x x}^{2} u_{0}(x)\right)^{2}}{2}+\Phi\left(u_{0}(x)\right)\right) d x=E[u](0) .
\end{aligned}
$$

We remind that

$$
h \in \partial \Phi(u)
$$

means that $h:[0, \infty) \times[0, L] \rightarrow \mathbb{R}$ satisfies

$$
h_{u}(t, x)\left\{\begin{array}{lr}
=\Phi^{\prime}(u(t, x)), & \text { if }|u(t, x)|<1, \\
=0, & \text { if }|u(t, x)|>1, \\
\in\left[0, \Phi^{\prime}\left(1^{-}\right)\right], & \text {if } u(t, x)=1, \\
\in\left[\Phi^{\prime}\left(-1^{+}\right), 0\right], & \text { if } u(t, x)=-1 .
\end{array}\right.
$$

Let us state the following theorem asserting the existence of dissipative solutions.

Theorem 3.2. (Existence) If $\Phi$ satisfies (H.1) and $u_{0}, u_{1}$ satisfy (H.2), then problem (2.1) admits a dissipative solution in the sense of Definition 3.1.

The following result provides a sufficient condition ruling the non-detachment of dissipative solutions in dependence on the initial data. 
Theorem 3.3. Let $u$ be a dissipative solution of (2.1). If

$$
\left\|u_{0}\right\|_{L^{\infty}(0, L)}<1 \quad \text { and } \quad E[u](0)<\frac{4 \kappa \mu}{3 \vee 2 \kappa}(\leq 2 \mu)
$$

where $\kappa$ is defined in (H.1), then

$$
\|u\|_{L^{\infty}((0, \infty) \times(0, L))}<1 .
$$

The long time behavior is a very subtle problem for evolutionary partial differential equations, so by restricting the focus on bounded dissipative solutions, we are able to prove the following statement.

Theorem 3.4. (Long time behavior) Let $u$ be a dissipative solution of (2.1) and $\left\{t_{n}\right\}_{n \in \mathbb{N}} \subset(0, \infty)$ such that $t_{n} \rightarrow \infty$. If

$$
u \in L^{\infty}((0, \infty) \times(0, L))
$$

then there exist a subsequence $\left\{t_{n_{k}}\right\}_{k \in \mathbb{N}}$ and two constants $a, b \in \mathbb{R}$ such that

$$
u\left(t_{n_{k}}, \cdot\right) \rightarrow u_{\infty} \quad \text { weakly in } H^{2}(0, L) \text { as } k \rightarrow \infty
$$

where

$$
u_{\infty}(x)=a x+b .
$$

Moreover, only one of the following statements can occur

$$
\begin{aligned}
u_{\infty} & \equiv 0, \\
u_{\infty}(x) & \geq 1 \text { for every } x \in[0, L], \\
u_{\infty}(x) & \leq-1 \text { for every } x \in[0, L] .
\end{aligned}
$$

The question at the basis of the last subsequent result can be formulated as follows: How the nonlinearity characterizing the forcing term $\Phi^{\prime}$ affects the evolution problem? We retain that Gérard's linearization condition provides a precise mathematical tool to answer to the previous rather vague question. Indeed the absence of further energy concentrations or oscillations due to the nonlinearity, suspected to arise in correspondence of the attachment-detachment process, constitutes an interesting property in itself, also considering the nonuniqueness of the solutions $\left\{u_{n}\right\}_{n}$ below.

Theorem 3.5. (Linearization property) Let $\left\{u_{0, n}\right\}_{n} \subset H^{2}(0, L),\left\{u_{1, n}\right\}_{n} \subset L^{2}(0, L)$, $\left\{u_{n}\right\}_{n}$ be a sequence of dissipative solutions of (2.1) in correspondence of such initial data, and $v_{n}$ be the dissipative solution of the linearized problem

$$
\begin{cases}\rho \partial_{t t}^{2} v_{n}=-\mu \partial_{x x x x}^{4} v_{n} & t>0,0<x<L, \\ \partial_{x x}^{2} v_{n}(t, 0)=\partial_{x x}^{2} v_{n}(t, L)=0 & t>0, \\ \partial_{x x x}^{3} v_{n}(t, 0)=\partial_{x x x}^{3} v_{n}(t, L)=0 & t>0 \\ v_{n}(0, x)=u_{0, n}(x) & 0<x<L \\ \partial_{t} v_{n}(0, x)=u_{1, n}(x) & 0<x<L .\end{cases}
$$


If

$$
\begin{aligned}
& u_{0, n} \rightarrow 0 \text { weakly in } H^{2}(0, L), \\
& u_{1, n} \rightarrow 0 \text { weakly in } L^{2}(0, L), \\
& \limsup _{n} \int_{0}^{L}\left(\frac{\rho u_{1, n}^{2}+\mu\left(\partial_{x x}^{2} u_{0, n}\right)^{2}}{2}\right) d x<\frac{4 \kappa \mu}{3 \vee 2 \kappa}(\leq 2 \mu),
\end{aligned}
$$

where $\kappa$ is defined in (H.1), then the following linearization condition holds true

$$
\left\|\partial_{t}\left(u_{n}-v_{n}\right)\right\|_{L^{\infty}\left(0, T ; L^{2}(0, L)\right)}+\left\|\partial_{x x}^{2}\left(u_{n}-v_{n}\right)\right\|_{L^{\infty}\left(0, T ; L^{2}(0, L)\right)} \rightarrow 0
$$

for every $T \geq 0$ as $n \rightarrow \infty$.

\section{Existence of Dissipative Solutions}

This section is dedicated to the proof of Theorem 3.2.

Our argument is based on the approximation of the Neumann problem (2.1) with a sequence of Neumann problems (4.1) characterized by smooth source terms and smooth initial data. More precisely, let $\left\{u_{0, n}\right\}_{n \in \mathbb{N}},\left\{u_{1, n}\right\}_{n \in \mathbb{N}} \subset C^{\infty}([0, L])$, $\left\{\Phi_{n}\right\}_{n \in \mathbb{N}} \subset C^{\infty}(\mathbb{R})$, for every $n \in \mathbb{N}$ consider the approximating problems

$$
\begin{cases}\rho \partial_{t t}^{2} u_{n}=-\mu \partial_{x x x x}^{4} u_{n}-\Phi_{n}^{\prime}\left(u_{n}\right) & t>0,0<x<L, \\ \partial_{x x}^{2} u_{n}(t, 0)=\partial_{x x}^{2} u_{n}(t, L)=0 & t>0, \\ \partial_{x x x}^{3} u_{n}(t, 0)=\partial_{x x x}^{3} u_{n}(t, L)=0 & t>0, \\ u_{n}(0, x)=u_{0, n}(x) & 0<x<L, \\ \partial_{t} u_{n}(0, x)=u_{1, n}(x) & 0<x<L,\end{cases}
$$

where $\left\{u_{0, n}\right\}_{n \in \mathbb{N}},\left\{u_{1, n}\right\}_{n \in \mathbb{N}},\left\{\Phi_{n}\right\}_{n \in \mathbb{N}}$ are sequences of smooth approximations of $u_{0}, u_{1}$, and $\Phi$ respectively, i.e. they satisfy the following requirements

$u_{0, n} \rightarrow u_{0} \quad$ in $H^{1}(0, L), \quad u_{1, n} \rightarrow u_{1} \quad$ in $L^{2}(0, L), \quad \Phi_{n} \rightarrow \Phi \quad$ uniformly in $\mathbb{R}$, $\Phi_{n}^{\prime} \rightarrow \Phi^{\prime} \quad$ pointwise and uniformly in $\mathbb{R} \backslash((-1-\varepsilon,-1+\varepsilon) \cup(1-\varepsilon, 1+\varepsilon))$ for every $\varepsilon$, $|u| \geq 1+\varepsilon \Rightarrow \Phi_{n}^{\prime}(u)=0, \quad \varepsilon>0, n \in \mathbb{N}$,

$\left\|u_{0, n}\right\|_{H^{1}(0, L)} \leq C, \quad\left\|u_{1, n}\right\|_{L^{2}(0, L)} \leq C, \quad 0 \leq \Phi_{n}, \Phi_{n}^{\prime} \leq C, \quad n \in \mathbb{N}$, $u_{0, n}^{\prime \prime}(0)=u_{0, n}^{\prime \prime}(L)=u_{1, n}(0)=u_{1, n}(L)=0, \quad n \in \mathbb{N}$,

where $C$ is a positive constant which does not depend on $n$.

For any $n \in \mathbb{N},(4.1)$ admits a classical solution for short time thanks to the Cauchy-Kowaleskaya Theorem (see [14]). Furthermore, for such a problem, solutions are indeed global in time thanks to the following results. Let $u_{n}$ be the unique classical solution to (4.1).

Lemma 4.1 (Energy conservation). Classical solution to (4.1) preserves energy. 
Proof. Set $E_{n}:=E\left[u_{n}\right]$ the energy corresponding to $u_{n}$. We have to prove that the function $t \mapsto E_{n}(t)$ is constant (with constant value $E_{n}(0)$ ). Indeed, we have that

$$
\begin{aligned}
E_{n}^{\prime}(t) & =\frac{d}{d t} \int_{0}^{L}\left(\frac{\rho\left(\partial_{t} u_{n}\right)^{2}+\mu\left(\partial_{x x}^{2} u_{n}\right)^{2}}{2}+\Phi_{n}\left(u_{n}\right)\right) d x \\
& =\int_{0}^{L}\left(\rho \partial_{t} u_{n} \partial_{t t}^{2} u_{n}+\mu \partial_{x x}^{2} u_{n} \partial_{t x x}^{3} u_{n}+\Phi_{n}^{\prime}\left(u_{n}\right) \partial_{t} u_{n}\right) d x \\
& =\int_{0}^{L} \partial_{t} u_{n} \underbrace{\left(\rho \partial_{t t}^{2} u_{n}+\mu \partial_{x x x x}^{4} u_{n}+\Phi_{n}^{\prime}\left(u_{n}\right)\right)}_{=0} d x=0 .
\end{aligned}
$$

As a consequence of energy conservation, since the functions $\Phi_{n}$ are positive, we have the following boundedness result.

Corollary 4.2. The sequences $\left\{\partial_{t} u_{n}\right\}_{n \in \mathbb{N}}$ and $\left\{\partial_{x x}^{2} u_{n}\right\}_{n \in \mathbb{N}}$ are bounded in $L^{\infty}\left(0, \infty ; L^{2}(0, L)\right)$.

Lemma 4.3 ( $L^{2}$ estimate). The sequence $\left\{u_{n}\right\}_{n \in \mathbb{N}}$ is bounded in $L^{\infty}\left(0, T ; L^{2}(0, L)\right)$, for every $T>0$.

Proof. Using the Hölder inequality

$$
\begin{aligned}
\int_{0}^{L} u_{n}^{2}(t, x) d x & =\int_{0}^{L}\left(u_{0, n}(x)+\int_{0}^{t} \partial_{s} u_{n}(s, x) d s\right)^{2} d x \\
& \leq 2 \int_{0}^{L} u_{0, n}^{2}(x) d x+2 \int_{0}^{L}\left(\int_{0}^{t}\left|\partial_{s} u_{n}(s, x)\right| d s\right)^{2} d x \\
& \leq 2 \int_{0}^{L} u_{0, n}^{2}(x) d x+2 t \int_{0}^{t} \int_{0}^{L}\left(\partial_{s} u_{n}(s, x)\right)^{2} d x d s \\
& \leq 2 \int_{0}^{L} u_{0, n}^{2}(x) d x+2 t^{2} \sup _{s \geq 0} \int_{0}^{L}\left(\partial_{s} u_{n}(s, x)\right)^{2} d x
\end{aligned}
$$

the claim follows from Corollary 4.2.

The following result follows from Corollary 4.2 by a straightforward application of Gagliardo-Nirenberg Interpolation Inequality (see for instance [12, Theorem at page 125]).

Lemma 4.4. ( $H^{1}$ estimate) The sequence $\left\{\partial_{x} u_{n}\right\}_{n \in \mathbb{N}}$ is bounded in $L^{\infty}\left(0, T ; L^{2}(0, L)\right)$, for every $T>0$.

Lemma 4.5. ( $L^{\infty}$ estimate) The sequence $\left\{u_{n}\right\}_{n \in \mathbb{N}}$ is bounded in $L^{\infty}((0, T) \times(0, L))$, for every $T>0$.

Proof. Fix $0<t<T$. Lemmas 4.1 and 4.3 and Corollary 4.2 imply that $\left\{u_{n}\right\}_{n \in \mathbb{N}}$ is bounded in $L^{\infty}\left(0, T ; H^{1}(0, L)\right)$. Since $H^{1}(0, L) \subset L^{\infty}(0, L)$ we have

$$
\begin{aligned}
\left|u_{n}(t, x)\right| & \leq\left\|u_{n}(t, \cdot)\right\|_{L^{\infty}(0, L)} \leq c\left\|u_{n}(t, \cdot)\right\|_{H^{1}(0, L)} \\
& \leq c\left\|u_{n}\right\|_{L^{\infty}\left(0, T ; H^{1}(0, L)\right)}, \quad(t, x) \in(0, T) \times(0, L),
\end{aligned}
$$


for some constant $c>0$ depending only on $L$. Therefore

$$
\left\|u_{n}\right\|_{L^{\infty}((0, T) \times(0, L))} \leq c\left\|u_{n}\right\|_{L^{\infty}\left(0, T ; H^{1}(0, L)\right)},
$$

that gives the claim.

Lemma 4.6 (Space Lipschitz estimate). The sequence $\left\{\partial_{x} u_{n}\right\}_{n \in \mathbb{N}}$ is bounded in $L^{\infty}((0, T) \times(0, L))$, for every $T>0$.

Proof. Fix $0<t<T$ and $0<x<L$. Lemmas 4.1, 4.3, and 4.4 imply that $\left\{u_{n}\right\}_{n \in \mathbb{N}}$ is bounded in $L^{\infty}\left(0, T ; H^{2}(0, L)\right)$. Since $H^{1}(0, L) \subset L^{\infty}(0, L)$ we have, for every $(t, x) \in(0, T) \times(0, L)$,

$$
\begin{aligned}
\left|\partial_{x} u_{n}(t, x)\right| & \leq\left\|\partial_{x} u_{n}(t, \cdot)\right\|_{L^{\infty}(0, L)} \\
& \leq c\left\|\partial_{x} u_{n}(t, \cdot)\right\|_{H^{1}(0, L)} \leq c\left\|u_{n}(t, \cdot)\right\|_{H^{2}(0, L)} \\
& \leq c\left\|u_{n}\right\|_{L^{\infty}\left(0, T ; H^{2}(0, L)\right)},
\end{aligned}
$$

for some constant $c>0$ depending only on $L$. Therefore

$$
\left\|\partial_{x} u_{n}\right\|_{L^{\infty}((0, T) \times(0, L))} \leq c\left\|u_{n}\right\|_{L^{\infty}\left(0, T ; H^{2}(0, L)\right)},
$$

that gives the claim.

Proof of Theorem 3.2. Thanks to Lemmas 4.1, 4.3 and [13, Theorem 5] there exists a function $u$ satisfying items $(i)$ and $(i i)$ in Definition 3.1 and a function $h_{u} \in$ $L^{\infty}((0, T) \times(0, L)), h_{u} \in \partial \Phi(u)$, such that, passing to a subsequence,

$$
\begin{aligned}
u_{n} \rightarrow u & \text { in } H^{1}((0, T) \times(0, L)) \text { and in } L^{2}\left(0, T ; H^{2}(0, L)\right), \text { for each } T \geq 0, \\
u_{n} \rightarrow u & \text { in } L^{\infty}((0, T) \times(0, L)), \text { for each } T \geq 0, \\
\Phi_{n}^{\prime}\left(u_{n}\right) \rightarrow h_{u} & \text { in } L^{p}((0, T) \times(0, L)), \text { for each } T \geq 0 \text { and } 1 \leq p<\infty .
\end{aligned}
$$

We have yet to verify that $u$ is a weak solution of (2.1) i.e. Definition 3.1-item (iii). Let $\varphi \in C^{\infty}\left(\mathbb{R}^{2}\right)$ be a test function with compact support, since $u_{n}$ is a solution to (4.1), we have that for every $n$

$$
\begin{aligned}
\int_{0}^{\infty} \int_{0}^{L} & \left(\rho u_{n} \partial_{t t}^{2} \varphi+\mu \partial_{x x}^{2} u_{n} \partial_{x x}^{2} \varphi+\Phi_{n}^{\prime}\left(u_{n}\right) \varphi\right) d x d t \\
& -\int_{0}^{L} \rho u_{1, n}(x) \varphi(0, x) d x+\int_{0}^{L} \rho u_{0, n}(x) \partial_{t} \varphi(0, x) d x=0 .
\end{aligned}
$$

Then, by taking the limit as $n \rightarrow \infty$, (3.1) follows by using (4.2) and (4.3).

Finally, due to (4.2) and (4.3) we have

$$
\begin{aligned}
\partial_{t} u_{n} \rightarrow \partial_{t} u & \text { in } L^{p}\left(0, T ; L^{2}(0, L)\right), \text { for each } T \geq 0 \text { and } 1 \leq p<\infty, \\
\partial_{x x}^{2} u_{n} \rightarrow \partial_{x x}^{2} u & \text { in } L^{p}\left(0, T ; L^{2}(0, L)\right), \text { for each } T \geq 0 \text { and } 1 \leq p<\infty, \\
\Phi_{n}\left(u_{n}\right) \rightarrow \Phi(u) & \text { in } L^{\infty}((0, T) \times(0, L)), \text { for each } T \geq 0 .
\end{aligned}
$$

Therefore, Definition 3.1-item (iv) follows by the lower semicontinuity of the $L^{2}$ norm with respect to the weak convergence by taking into account Lemma 4.1. 


\section{Adhesive States}

This section is dedicated to the proof of Theorem 3.3.

Proof of Theorem 3.3. Since $u$ is continuous by (3.3) there exists $\tau>0$ such that in the short time interval $[0, \tau]$ we have

$$
|u(t, x)|<1, \quad(t, x) \in[0, \tau] \times[0, L] .
$$

So we can define $\tau^{*}$ as follows

$$
\tau^{*}=\sup \{\tau>0|| u(t, x) \mid<1 \text { for all }(t, x) \in[0, \tau] \times[0, L]\} .
$$

We claim that

$$
\tau^{*}=\infty
$$

Observe that, due to (H.1),

$$
\Phi(u(t, x)) \geq \kappa u^{2}(t, x), \quad(t, x) \in\left[0, \tau^{*}\right) \times[0, L] .
$$

Therefore

$$
E[u](t) \geq \int_{0}^{L}\left(\frac{\rho\left(\partial_{t} u(t, x)\right)^{2}+\mu\left(\partial_{x x}^{2} u(t, x)\right)^{2}}{2}+\kappa(u(t, x))^{2}\right) d x, \quad t \in\left[0, \tau^{*}\right),
$$

and, in particular,

$$
E[u](t) \geq \frac{\mu\left\|\partial_{x x}^{2} u(t, \cdot)\right\|_{L^{2}(0, L)}^{2}}{2}+\kappa\|u(t, \cdot)\|_{L^{2}(0, L)}^{2}, \quad t \in\left[0, \tau^{*}\right) .
$$

Since $u$ is dissipative, using the Sobolev embedding $H^{1}(0, L) \subset L^{\infty}(0, L)$ (see $[6$, Theorem 8.5]) and Lemma 4.4, we have from every $t \in\left[0, \tau^{*}\right)$

$$
\begin{aligned}
\|u(t, \cdot)\|_{L^{\infty}(0, L)}^{2} & \leq \frac{\|u(t, \cdot)\|_{H^{1}(0, L)}^{2}}{2}=\frac{\|u(t, \cdot)\|_{L^{2}(0, L)}^{2}+\left\|\partial_{x} u(t, \cdot)\right\|_{L^{2}(0, L)}^{2}}{2} \\
& \leq \frac{3}{4}\|u(t, \cdot)\|_{L^{2}(0, L)}^{2}+\frac{\left\|\partial_{x x}^{2} u(t, \cdot)\right\|_{L^{2}(0, L)}^{2}}{4} \\
& \leq\left(\frac{3}{4 \kappa} \vee \frac{1}{2}\right)\left(\frac{\left\|\partial_{x x}^{2} u(t, \cdot)\right\|_{L^{2}(0, L)}^{2}}{2}+\kappa\|u(t, \cdot)\|_{L^{2}(0, L)}^{2}\right) \\
& \leq \frac{1}{\mu}\left(\frac{3}{4 \kappa} \vee \frac{1}{2}\right) E[u](t) \leq \frac{1}{\mu}\left(\frac{3}{4 \kappa} \vee \frac{1}{2}\right) E[u](0)<1,
\end{aligned}
$$

(where the last inequality holds thanks to (3.3)) that proves (5.1).

\section{Long Time Behavior}

This section is dedicated to the proof of Theorem 3.4.

Let $u$ be a dissipative solution of (2.1) satisfying (3.5).

STEP 1. We begin by deducing the effective asymptotic problem.

Consider the functions

$$
u_{\tau}(t, x)=u(\tau t, x), \quad \tau>0, t \geq 0, x \in[0, L] .
$$


$u_{\tau}$ is a dissipative solution of the initial boundary value problem

$$
\begin{cases}\frac{\rho \partial_{t t}^{2} u_{\tau}}{\tau^{2}}=-\mu \partial_{x x x x}^{4} u_{\tau}-\Phi^{\prime}\left(u_{\tau}\right) & t>0,0<x<L, \\ \partial_{x x}^{2} u_{\tau}(t, 0)=\partial_{x x}^{2} u_{\tau}(t, L)=0 & t>0, \\ \partial_{x x x}^{3} u_{\tau}(t, 0)=\partial_{x x x}^{3} u_{\tau}(t, L)=0 & t>0, \\ u_{\tau}(0, x)=u_{0}(x) & 0<x<L, \\ \partial_{t} u_{\tau}(0, x)=\tau u_{1}(x) & 0<x<L,\end{cases}
$$

in the sense of Definition 3.1, namely

(iii) for every test function $\varphi \in C^{\infty}\left(\mathbb{R}^{2}\right)$ with compact support

$$
\int_{0}^{\infty} \int_{0}^{L}\left(-\frac{\rho \partial_{t} u_{\tau}}{\tau^{2}} \partial_{t} \varphi+\mu \partial_{x x}^{2} u_{\tau} \partial_{x x}^{2} \varphi+h_{\tau} \varphi\right) d t d x-\int_{0}^{L} \frac{\rho u_{1}(x)}{\tau} \varphi(0, x) d x=0
$$

where $h_{\tau} \in \partial \Phi\left(u_{\tau}\right)$, that is the subdifferential of $\Phi$ at $u_{\tau}$;

(iv) $u_{\tau}$ may dissipate energy, i.e. for almost every $t>0$ :

$$
\begin{gathered}
\int_{0}^{L}\left(\frac{\rho\left(\partial_{t} u_{\tau}(t, x)\right)^{2}}{2 \tau^{2}}+\frac{\mu\left(\partial_{x x}^{2} u_{\tau}(t, x)\right)^{2}}{2}+\Phi\left(u_{\tau}(t, x)\right)\right) d x \\
\leq \int_{0}^{L}\left(\frac{\rho\left(u_{1}(x)\right)^{2}}{2}+\frac{\mu\left(\partial_{x x}^{2} u_{0}(x)\right)^{2}}{2}+\Phi\left(u_{0}(x)\right)\right) d x
\end{gathered}
$$

Thanks to (H.1), (3.5), and (6.3),

$$
\begin{aligned}
& \left\{u_{\tau}\right\}_{\tau>0} \text { is bounded in } L^{\infty}\left(0, \infty ; H^{2}(0, L)\right), \\
& \left\{h_{\tau}\right\}_{\tau>0} \text { is bounded in } L^{\infty}((0, \infty) \times(0, L)),
\end{aligned}
$$

there exists two functions $U \in L^{\infty}\left(0, \infty ; H^{2}(0, L)\right), H \in L^{\infty}((0, \infty) \times(0, L))$ such that, passing to a subsequence,

$$
\begin{array}{ll}
u_{\tau} \stackrel{\star}{\rightarrow} U & \text { weakly }-\star \text { in } L_{\text {loc }}^{\infty}((0, \infty) \times(0, L)) \text { as } \tau \rightarrow \infty, \\
h_{\tau} \stackrel{\star}{\rightarrow} H \quad \text { weakly }-\star \text { in } L_{\text {loc }}^{\infty}((0, \infty) \times(0, L)) \text { as } \tau \rightarrow \infty .
\end{array}
$$

Using (6.3)

$$
\left\{\partial_{t} u_{\tau} / \tau\right\}_{n \in \mathbb{N}} \text { is bounded in } L^{\infty}\left(0, \infty ; L^{2}(0, L)\right),
$$

therefore as $\tau \rightarrow \infty$ in (6.2) we get

$$
\int_{0}^{\infty} \int_{0}^{L}\left(\mu \partial_{x x}^{2} U \partial_{x x}^{2} \varphi+H \varphi\right) d t d x=0
$$

namely $U=U(x), H=H(x)$ and the effective asymptotic problem is

$$
\begin{cases}-\mu \partial_{x x x x}^{4} U=H, & 0<x<L, \\ \partial_{x x}^{2} U(0)=\partial_{x x}^{2} U(L)=0, & \\ \partial_{x x x}^{3} U(0)=\partial_{x x x}^{3} U(L)=0 . & \end{cases}
$$


STEP 2. We exploit more subtle characterizations of the limit functions $U$ and $H$. To this aim we fix a sequence $\left\{t_{n}\right\}_{n \in \mathbb{N}} \subset(0, \infty)$ such that $t_{n} \rightarrow \infty$ and study the convergence of the sequence

$$
\left\{u\left(t_{n}, \cdot\right)\right\}_{n \in \mathbb{N}} .
$$

Since we have the dissipation inequality (3.2) and the assumption (3.5), we gain

$$
\begin{aligned}
& \left\{u\left(t_{n}, \cdot\right)\right\}_{n \in \mathbb{N}} \text { is bounded in } H^{2}(0, L), \\
& \left\{h_{u}\left(t_{n}, \cdot\right)\right\}_{n \in \mathbb{N}} \text { is bounded in } L^{\infty}(0, L) .
\end{aligned}
$$

Therefore there exist two functions $u_{\infty} \in H^{2}(0, L), h_{\infty} \in L^{\infty}(0, L)$ such that passing to a subsequence

$$
\begin{aligned}
& u\left(t_{n}, \cdot\right) \rightarrow u_{\infty} \quad \text { weakly in } H^{2}(0, L) \text { as } n \rightarrow \infty, \\
& u\left(t_{n}, \cdot\right) \rightarrow u_{\infty} \quad \text { a.e. in }(0, L) \text { as } n \rightarrow \infty, \\
& h_{u}\left(t_{n}, \cdot\right) \stackrel{\star}{\rightarrow} h_{\infty} \quad \text { weakly }-\star \text { in } L^{\infty}(0, L) \text { as } n \rightarrow \infty .
\end{aligned}
$$

Due to the result in STEP 1, we know that the functions $u_{\infty}$ and $h_{\infty}$ must satisfy the effective problem

$$
\begin{cases}-\mu \partial_{x x x x}^{4} u_{\infty}=h_{\infty}, & 0<x<L \\ \partial_{x x}^{2} u_{\infty}(0)=\partial_{x x}^{2} u_{\infty}(L)=0 & \\ \partial_{x x x}^{3} u_{\infty}(0)=\partial_{x x x}^{3} u_{\infty}(L)=0 . & \end{cases}
$$

Moreover, by (6.6) we have also that

$$
h_{\infty} \in \partial \Phi^{\prime}\left(u_{\infty}\right)
$$

By multiplying $(6.7)$ by $u_{\infty}$, integrating over $(0, L)$, and recalling $(6.8)$, we get

$$
\int_{0}^{L} \mu\left(\partial_{x x}^{2} u_{\infty}\right)^{2} d x=-\int_{0}^{L} h_{\infty} u_{\infty} d x \leq 0
$$

SO

$$
\partial_{x x}^{2} u_{\infty} \equiv 0
$$

Therefore, we can conclude that (3.6) holds.

Using (3.6) in (6.7) we have also that

$$
h_{\infty} \equiv 0,
$$

hence, due to (6.8), only one within (3.7), (3.8), (3.9) can occur.

\section{Linearization Property}

Proof of Theorem 3.5. Thanks to (3.11)

$$
u_{0, n} \rightarrow 0 \quad \text { uniformly in }[0, L] .
$$

Therefore, using also (3.13), the assumptions of Theorem 3.3 are fulfilled, hence

$$
\left\|u_{n}\right\|_{L^{\infty}((0, \infty) \times(0, L))}<1 .
$$

In addition, the function

$$
w_{n}=u_{n}-v_{n}
$$


is a dissipative solution of

$$
\begin{cases}\rho \partial_{t t}^{2} w_{n}=-\mu \partial_{x x x x}^{4} w_{n}-\Phi^{\prime}\left(u_{n}\right) & t>0,0<x<L, \\ \partial_{x x}^{2} w_{n}(t, 0)=\partial_{x x}^{2} w_{n}(t, L)=0 & t>0, \\ \partial_{x x x}^{3} w_{n}(t, 0)=\partial_{x x x}^{3} w_{n}(t, L)=0 & t>0, \\ w_{n}(0, x)=\partial_{t} w_{n}(0, x)=0 & 0<x<L .\end{cases}
$$

Multiplying (7.2) by $\partial_{t} w_{n}$ we gain

$$
\begin{aligned}
\frac{d}{d t} \int_{0}^{L} \frac{\rho\left(\partial_{t} w_{n}\right)^{2}+\mu\left(\partial_{x x}^{2} w_{n}\right)^{2}}{2} d x & =\int_{0}^{L} \Phi^{\prime}\left(u_{n}\right) \partial_{t} w_{n} d x \\
& \leq \frac{1}{2 \rho} \int_{0}^{L} \Phi^{\prime}\left(u_{n}\right)^{2} d x+\int_{0}^{L} \frac{\rho\left(\partial_{t} w_{n}\right)^{2}}{2} d x \\
& \leq \frac{1}{2 \rho} \int_{0}^{L} \Phi^{\prime}\left(u_{n}\right)^{2} d x+\int_{0}^{L} \frac{\rho\left(\partial_{t} w_{n}\right)^{2}+\mu\left(\partial_{x x}^{2} w_{n}\right)^{2}}{2} d x
\end{aligned}
$$

and applying the Gronwall Lemma

$$
\int_{0}^{L} \frac{\rho\left(\partial_{t} w_{n}(t, x)\right)^{2}+\mu\left(\partial_{x x}^{2} w_{n}(t, x)\right)^{2}}{2} d x \leq \frac{1}{2 \rho} \int_{0}^{t} \int_{0}^{L} e^{t-s} \Phi^{\prime}\left(u_{n}(s, x)\right)^{2} d s d x .
$$

Thanks to Theorem 3.2 and [13, Theorem 5], and (7.1) there exists a function $u$ satisfying items $(i)$ and $(i i)$ in Definition 3.1 such that, passing to a subsequence,

$\|u\|_{L^{\infty}((0, \infty) \times(0, L))} \leq 1$,

$u_{n} \rightarrow u$ in $H^{1}((0, T) \times(0, L))$ and in $L^{2}\left(0, T ; H^{2}(0, L)\right)$, for each $T \geq 0$,

$u_{n} \rightarrow u$ in $L^{\infty}((0, T) \times(0, L))$, for each $T \geq 0$,

$\Phi^{\prime}\left(u_{n}\right) \rightarrow \Phi^{\prime}(u)$ in $L^{p}((0, T) \times(0, L))$, for each $T \geq 0$ and $1 \leq p<\infty$.

Therefore $u$ is a distributional solution of

$$
\begin{cases}\rho \partial_{t t}^{2} u=-\mu \partial_{x x x x}^{4} u-\Phi^{\prime}(u) & t>0,0<x<L, \\ \partial_{x x}^{2} u(t, 0)=\partial_{x x}^{2} u(t, L)=0 & t>0, \\ \partial_{x x x}^{3} u(t, 0)=\partial_{x x x}^{3} u(t, L)=0 & t>0, \\ u(0, x)=\partial_{t} u(0, x)=0 & 0<x<L .\end{cases}
$$

Since $u$ takes values in $[-1,1]$ and $\Phi$ is $C^{2}$ therein we can differentiate (7.5) and get

$$
\rho \partial_{t t t}^{3} u=-\mu \partial_{t x x x x}^{4} u-\Phi^{\prime \prime}(u) \partial_{t} u .
$$

Multiplying by $\partial_{t t}^{2} u$, using (H.1) and (ii) in Definition 3.1 through a regularization argument we get

$$
\begin{aligned}
\frac{d}{d t} \int_{0}^{L} \frac{\rho\left(\partial_{t t}^{2} u\right)^{2}+\mu\left(\partial_{t x x}^{3} u\right)^{2}}{2} d x & =\int_{0}^{L} \Phi^{\prime \prime}(u) \partial_{t} u \partial_{t t}^{2} u d x \\
& \leq \frac{1}{2 \rho} \int_{0}^{L} \Phi^{\prime \prime}(u)^{2}\left(\partial_{t} u\right)^{2} d x+\int_{0}^{L} \frac{\rho\left(\partial_{t t}^{2} u\right)^{2}}{2} d x \\
& \leq c+\int_{0}^{L} \frac{\rho\left(\partial_{t t}^{2} u\right)^{2}+\mu\left(\partial_{t x x}^{3} u\right)^{2}}{2} d x
\end{aligned}
$$


Thanks to the Gronwall Lemma

$$
\int_{0}^{L} \frac{\rho\left(\partial_{t t}^{2} u(t, x)\right)^{2}+\mu\left(\partial_{t x x}^{3} u(t, x)\right)^{2}}{2} d x \leq c\left(e^{t}-1\right), \quad t \geq 0 .
$$

As a consequence $u$ is an energy preserving solution of (7.5) and then it must be the trivial one. Eventually, (7.3) concludes the proof.

\section{Non-uniqueness and Lack of Smoothness}

This section is devoted to exploit some qualitative properties of (2.1) through explicit analytical examples evidencing the lack of uniqueness and smoothness of solutions. A key mechanism ruling these phenomena relies in the transition between the two configurations induced by the discontinuity affecting the forcing term $\Phi^{\prime}$. In particular, the first two examples show the lack of uniqueness while the last one and the numerical experiments enlighten the occurrences of lack of smoothness.

Example 8.1. Let $\varepsilon>0$ and set $\rho=\mu=1$. Consider the function

$$
\Phi_{\varepsilon}(u)= \begin{cases}\frac{2-\varepsilon}{2} u^{2}, & \text { if }|u| \leq 1, \\ \frac{2-\varepsilon}{\varepsilon}\left((1+\varepsilon)\left(u-\frac{1}{2}\right)-\frac{u^{2}}{2}\right), & \text { if } 1 \leq u \leq 1+\varepsilon, \\ \frac{\varepsilon-2}{\varepsilon}\left((1+\varepsilon)\left(u+\frac{1}{2}\right)+\frac{u^{2}}{2}\right), & \text { if }-1-\varepsilon \leq u \leq-1, \\ \frac{(2-\varepsilon)(1+\varepsilon)}{2}, & \text { if }|u| \geq 1+\varepsilon .\end{cases}
$$

We have

$$
\Phi_{\varepsilon}^{\prime}(u)= \begin{cases}(2-\varepsilon) u, & \text { if }|u| \leq 1 \\ \frac{2-\varepsilon}{\varepsilon}(1+\varepsilon-u), & \text { if } 1 \leq u \leq 1+\varepsilon \\ \frac{\varepsilon-2}{\varepsilon}(1+\varepsilon+u), & \text { if }-1-\varepsilon \leq u \leq-1, \\ 0, & \text { if }|u| \geq 1+\varepsilon\end{cases}
$$

The functions

$$
u_{\varepsilon}(t, x)=(1-\varepsilon) \cos (\sqrt{2-\varepsilon} t), \quad v_{\varepsilon}(t, x)=1+\varepsilon
$$

solve

$$
\begin{aligned}
& \begin{cases}\partial_{t t}^{2} u_{\varepsilon}=-\partial_{x x x x}^{4} u_{\varepsilon}-\Phi_{\varepsilon}^{\prime}\left(u_{\varepsilon}\right), & t>0,0<x<L, \\
\partial_{x x}^{2} u_{\varepsilon}(t, 0)=\partial_{x x}^{2} u_{\varepsilon}(t, L)=0, & t>0, \\
\partial_{x x x}^{3} u_{\varepsilon}(t, 0)=\partial_{x x x}^{3} u_{\varepsilon}(t, L)=0, & t>0, \\
u_{\varepsilon}(0, x)=1-\varepsilon, & 0<x<L, \\
\partial_{t} u_{\varepsilon}(0, x)=0, & 0<x<L,\end{cases} \\
& \begin{cases}\partial_{t t}^{2} v_{\varepsilon}=-\partial_{x x x x}^{4} v_{\varepsilon}-\Phi_{\varepsilon}^{\prime}\left(v_{\varepsilon}\right), & t>0,0<x<L, \\
\partial_{x x}^{2} v_{\varepsilon}(t, 0)=\partial_{x x}^{2} v_{\varepsilon}(t, L)=0, & t>0, \\
\partial_{x x x}^{3} v_{\varepsilon}(t, 0)=\partial_{x x x}^{3} v_{\varepsilon}(t, L)=0, & t>0, \\
v_{\varepsilon}(0, x)=1+\varepsilon, & 0<x<L \\
\partial_{t} v_{\varepsilon}(0, x)=0, & 0<x<L\end{cases}
\end{aligned}
$$


As $\varepsilon \rightarrow 0$ we have

$$
u_{\varepsilon}(t, x) \rightarrow u(t, x)=\cos (\sqrt{2} t), \quad v_{\varepsilon}(t, x) \rightarrow v(t, x)=1,
$$

and $u$ and $v$ provides two different solutions of (2.1) in correspondence of the initial data

$$
u_{0}(x)=1, \quad u_{1}(x)=0 .
$$

The energies associated to (8.1) and (8.2) are

$$
\begin{aligned}
& E_{\varepsilon}\left[u_{\varepsilon}\right](t)=\int_{0}^{L}\left(\frac{\left(\partial_{t} u_{\varepsilon}(t, x)\right)^{2}+\left(\partial_{x x}^{2} u_{\varepsilon}(t, x)\right)^{2}}{2}+\Phi_{\varepsilon}\left(u_{\varepsilon}(t, x)\right)\right) d x=\frac{(2-\varepsilon)(1-\varepsilon)^{2}}{2} L, \\
& E_{\varepsilon}\left[v_{\varepsilon}\right](t)=\int_{0}^{L}\left(\frac{\left(\partial_{t} v_{\varepsilon}(t, x)\right)^{2}+\left(\partial_{x x}^{2} v_{\varepsilon}(t, x)\right)^{2}}{2}+\Phi_{\varepsilon}\left(v_{\varepsilon}(t, x)\right)\right) d x=\frac{(2-\varepsilon)(1+\varepsilon)}{2} L,
\end{aligned}
$$

respectively.

Example 8.2. For every $\varepsilon>0$, the solutions $u_{\varepsilon}$ and $v_{\varepsilon}$ of the two following problems

$$
\begin{aligned}
& \begin{cases}\partial_{t t}^{2} u_{\varepsilon}=-\partial_{x x x x}^{4} u_{\varepsilon}-\Phi^{\prime}\left(u_{\varepsilon}\right), & t>0,0<x<L, \\
\partial_{x x}^{2} u_{\varepsilon}(t, 0)=\partial_{x x}^{2} u_{\varepsilon}(t, L)=0, & t>0, \\
\partial_{x x x}^{3} u_{\varepsilon}(t, 0)=\partial_{x x x}^{3} u_{\varepsilon}(t, L)=0, & t>0, \\
u_{\varepsilon}(0, x)=1+\varepsilon, & 0<x<L, \\
\partial_{t} u_{\varepsilon}(0, x)=\varepsilon, & 0<x<L,\end{cases} \\
& \begin{cases}\partial_{t t}^{2} v_{\varepsilon}=-\partial_{x x x x}^{4} v_{\varepsilon}-\Phi^{\prime}\left(v_{\varepsilon}\right), & t>0,0<x<L, \\
\partial_{x x}^{2} v_{\varepsilon}(t, 0)=\partial_{x x}^{2} v_{\varepsilon}(t, L)=0, & t>0, \\
\partial_{x x x}^{3} v_{\varepsilon}(t, 0)=\partial_{x x x}^{3} v_{\varepsilon}(t, L)=0, & t>0, \\
v_{\varepsilon}(0, x)=1-\varepsilon, & 0<x<L, \\
\partial_{t} v_{\varepsilon}(0, x)=0, & 0<x<L,\end{cases}
\end{aligned}
$$

are

$$
u_{\varepsilon}(t, x)=\varepsilon t+1+\varepsilon, \quad v_{\varepsilon}(t, x)=(1-\varepsilon) \cos (\sqrt{2} t) .
$$

We have

$$
\begin{aligned}
& \left\|u_{\varepsilon}(0, \cdot)-v_{\varepsilon}(0, \cdot)\right\|_{L^{2}(0, L)}+\left\|\partial_{t} u_{\varepsilon}(0, \cdot)-\partial_{t} v_{\varepsilon}(0, \cdot)\right\|_{L^{2}(0, L)}=3 \varepsilon \sqrt{L}, \\
& \lim _{t \rightarrow \infty} u_{\varepsilon}(t, x)=\infty, \quad \limsup _{t \rightarrow \infty} v_{\varepsilon}(t, x)=1-\varepsilon
\end{aligned}
$$

Moreover, as $\varepsilon \rightarrow 0$,

$$
u_{\varepsilon}(t, x) \rightarrow 1, \quad v_{\varepsilon}(t, x) \rightarrow \cos (\sqrt{2} t) .
$$

The energies associated to (8.3) and (8.4) are

$$
\begin{aligned}
& E\left[u_{\varepsilon}\right](t)=\int_{0}^{L}\left(\frac{\left(\partial_{t} u_{\varepsilon}(t, x)\right)^{2}+\left(\partial_{x x}^{2} u_{\varepsilon}(t, x)\right)^{2}}{2}+\Phi\left(u_{\varepsilon}(t, x)\right)\right) d x=\frac{\varepsilon^{2}+2}{2} L, \\
& E\left[v_{\varepsilon}\right](t)=\int_{0}^{L}\left(\frac{\left(\partial_{t} v_{\varepsilon}(t, x)\right)^{2}+\left(\partial_{x x}^{2} v_{\varepsilon}(t, x)\right)^{2}}{2}+\Phi\left(v_{\varepsilon}(t, x)\right)\right) d x=(1-\varepsilon)^{2} L,
\end{aligned}
$$

respectively. 
Example 8.3. Consider the function

$$
u(t, x)= \begin{cases}\sqrt{2} \sin (\sqrt{2} t), & \text { if } 0 \leq t \leq \frac{\pi}{4 \sqrt{2}}, \\ \sqrt{2} t+1-\frac{\pi}{4}, & \text { if } t \geq \frac{\pi}{4 \sqrt{2}} .\end{cases}
$$

Clearly, $u$ solves the problem

$$
\begin{cases}\partial_{t t}^{2} u=-\partial_{x x x x}^{4} u-\Phi^{\prime}(u), & t>0, x \in(0, L), \\ \partial_{x x}^{2} u(t, 0)=\partial_{x x}^{2} u(t, L)=0, & t>0, \\ \partial_{x x x}^{3} u(t, 0)=\partial_{x x x}^{3} u(t, L)=0, & t>0, \\ u(0, x)=0, & x \in(0, L), \\ \partial_{t} u(0, x)=2, & x \in(0, L),\end{cases}
$$

but

$$
u \in C^{1}([0, \infty) \times[0, L]) \backslash C^{2}([0, \infty) \times[0, L]) .
$$

Indeed

$$
\begin{array}{cc}
\lim _{t \rightarrow \frac{\pi}{4 \sqrt{2}}-} u(t, x)=1, & \lim _{t \rightarrow \frac{\pi}{4 \sqrt{2}}+} u(t, x)=1, \\
\lim _{t \rightarrow \frac{\pi}{4 \sqrt{2}}-} \partial_{t} u(t, x)=\sqrt{2}, & \lim _{t \rightarrow \frac{\pi}{4 \sqrt{2}}+} \partial_{t} u(t, x)=\sqrt{2}, \\
\lim _{t \rightarrow \frac{\pi}{4 \sqrt{2}}-} \partial_{t t}^{2} u(t, x)=-2, & \lim _{t \rightarrow \frac{\pi}{4 \sqrt{2}}+} \partial_{t t}^{2} u(t, x)=0 .
\end{array}
$$

The energy associated to (8.5) is

$$
E[u](t)=\int_{0}^{L}\left(\frac{\left(\partial_{t} u(t, x)\right)^{2}+\left(\partial_{x} u(t, x)\right)^{2}}{2}+\Phi(u(t, x))\right) d x=2 L,
$$

for every $t \geq 0$.

Funding Open access funding provided by Politecnico di Bari within the CRUICARE Agreement.

Open Access. This article is licensed under a Creative Commons Attribution 4.0 International License, which permits use, sharing, adaptation, distribution and reproduction in any medium or format, as long as you give appropriate credit to the original author(s) and the source, provide a link to the Creative Commons licence, and indicate if changes were made. The images or other third party material in this article are included in the article's Creative Commons licence, unless indicated otherwise in a credit line to the material. If material is not included in the article's Creative Commons licence and your intended use is not permitted by statutory regulation or exceeds the permitted use, you will need to obtain permission directly from the copyright holder. To view a copy of this licence, visit http://creativecommons.org/licenses/by/4.0/.

\section{References}

[1] Burridge, R., Keller, J.B.: Peeling, slipping and cracking - some one-dimensional freeboundary problems in mechanics. SIAM Rev. 20(1), 31-61 (1978)

[2] Coclite, G.M., Fanizza, G., Maddalena, F.: Regularity and energy transfer for a nonlinear beam equation. Appl. Math. Lett. 115, 106959 (2021) 
[3] Coclite, G.M., Florio, G., Ligabò, M., Maddalena, F.: Nonlinear waves in adhesive strings. SIAM J. Appl. Math. 77(2), 347-360 (2017)

[4] Coclite, G.M., Florio, G., Ligabò, M., Maddalena, F.: Adhesion and debonding in a model of elastic string. Comput. Math. Appl. 78(6), 1897-1909 (2019)

[5] Gérard, P.: Oscillations of concentration effects in semilinear dispersive wave equations. J. Funct. Anal. 141(1), 60-98 (1996)

[6] Lieb, E.H., Loss, M.: Analysis, Graduate Studies in Mathematics, vol. 14, 2nd ed. American Mathematical Society, Providence (2001)

[7] Maddalena, F., Percivale, D.: Variational models for peeling problems. Interfaces Free Bound. 10(4), 503-516 (2008)

[8] Maddalena, F., Percivale, D., Puglisi, G., Truskinovsky, L.: Mechanics of reversible unzipping. Contin. Mech. Thermodyn. 21(4), 251-268 (2009)

[9] Maddalena, F., Percivale, D., Tomarelli, F.: Adhesive flexible material structures. Discrete Contin. Dyn. Syst. Ser. B 17(2), 553-574 (2012)

[10] Maddalena, F., Percivale, D., Tomarelli, F.: Local and non-local energies in adhesive interaction. IMA J. Appl. Math. 81(6), 1051-1075 (2016)

[11] Maddalena, F., Percivale, D., Tomarelli, F.: Elastic-brittle reinforcement of flexural structures. arXiv:2105.05077 (2021)

[12] Nirenberg, L.: On elliptic partial differential equations. Ann. Scuola Norm. Sup. Pisa Cl. Sci. (3) 13, 115-162 (1959)

[13] Simon, J.: Compact sets in the space $L^{p}(0, T ; B)$. Ann. Mat. Pura Appl. 4(146), 65-96 (1987)

[14] Taylor, M.E.: Partial Differential Equations I. Basic Theory, Applied Mathematical Sciences, vol. 115, 2nd ed. Springer, New York (2011)

G. M. Coclite, G. Devillanova and F. Maddalena

Dipartimento di Meccanica, Matematica e Management

Politecnico di Bari

Via E. Orabona 4

70125 Bari

Italy

e-mail: giuseppemaria.coclite@poliba.it

G. Devillanova

e-mail: giuseppe.devillanova@poliba.it

F. Maddalena

e-mail: francesco.maddalena@poliba.it

Received: May 25, 2021.

Accepted: September 14, 2021. 